\title{
Positive intratumoral chemokine (C-C motif) receptor 8 expression predicts high recurrence risk of post-operation clear- cell renal cell carcinoma patients
}

\author{
Qiang Fu ${ }^{1,}{ }^{*}$, Yuan Chang ${ }^{2,}$, Lin Zhou ${ }^{2}$, Huimin An ${ }^{3}$, Yu Zhu ${ }^{3}$, Le Xu ${ }^{4}$, Weijuan Zhang ${ }^{5}$ \\ and Jiejie $X \mathbf{u}^{1}$ \\ ${ }^{1}$ Department of Biochemistry and Molecular Biology, School of Basic Medical Sciences, Fudan University, Shanghai, China \\ 2 Department of Urology, Zhongshan Hospital, Fudan University, Shanghai, China \\ ${ }^{3}$ Department of Urology, Shanghai Cancer Center, Fudan University, Shanghai, China \\ ${ }^{4}$ Department of Urology, Ruijin Hospital, School of Medicine, Shanghai Jiaotong University, Shanghai, China \\ ${ }^{5}$ Department of Immunology, School of Basic Medical Sciences, Fudan University, Shanghai, China \\ * These authors have contributed equally to this work \\ Correspondence to: Weijuan Zhang, email: weijuanzhang@fudan.edu.cn
}

Jiejie Xu, email: jjxufdu@fudan.edu.cn

Keywords: chemokine (C-C motif) receptor 8, clear-cell renal cell carcinoma, prognostic factor, recurrence-free survival Received: September 08, 2015 Accepted: December 07, 2015

Published: December 24, 2015

\section{ABSTRACT}

Chemokine (C-C motif) receptor 8 (CCR8) could drive cancer progress through recruiting certain immune cells. Recent evidences revealed the chemotaxis of CCR8 ${ }^{+}$ human malignant tumor cells towards lymph node, and a significantly increased CCR8 expression in renal carcinomas patients. To assess the clinical association between CCR8 expression and the risk of post-surgery recurrence in patients with clear-cell renal cell carcinoma (CCRCC), we detected intratumoral CCR8 expression in 472 postnephrectomy CCRCC patients retrospectively enrolled. Positive CCR8 staining tumor cell occurred in $26.1 \%$ (123 of 472 ) non-metastatic ccRCC cases, and the positive expression was associated with increased risks of recurrence (Log-Rank $P<0.001)$. In multivariate analyses, CCR8 expression was identified as an independent prognostic factor $(P=0.008)$ and entered into a newly-built nomogram together with $T$ stage, Fuhrman grade, tumor size, necrosis and lymphovascular invasion. Calibration curves showed optimal agreement between predictions and observations, while its C-index was higher than that of Leibovich score for predicting recurrence-free survival (RFS) of localised RCC patients $(0.854$ vs 0.836 , respectively; $P=0.044)$. The practical prognostic nomogram model may help clinicians in decision making and design of clinical studies.

\section{INTRODUCTION}

Chemokine (C-C motif) receptor 8 (CCR8), one member of the $\mathrm{C}-\mathrm{C}$ motif chemokine receptor superfamily, was initially identified in activated T helper type 2 (Th2) cells as a receptor of CCL1/I-309 [1,2]. The CCL1CCR8 axis is involved in the pathological processes of various inflammatory diseases. This chaining is believed to mediate Th2 cell and Treg cell recruitment in allergic inflammation such as asthma and atopic dermatitis. CCR $8^{+}$ macrophages also play significant roles in postoperative peritoneal adhesion development [3]. The involvement of CCR8 in these diseases suggests that it is a unique modular in inflammatory/allergic responses by inducing tissue damage and remodeling. Recently some studies focusing on the behavior of CCR8 in carcinogenesis were conducted and have implicated an anti-apoptosis effect of CCR8 in lymphoma and T cell leukemia through an autocrine manner [4]. Unexpectedly, Suvendu et al reported that CCL1 produced by lymphatic endothelial cells could facilitates $\mathrm{CCR} 8^{+}$tumor cell entry into the open subcapular sinus as well as subsequent migration into the lymph node cortex [5]. These data identify a novel mechanism of regulation of tumor cells migration 
by virtue of CCR8. However, whether CCR8 plays a role in clinical recurrence of patients with solid tumors is not yet determined.

Renal cell carcinoma (RCC) represents 3 4\% of adult solid tumors. It is frequently diagnosed with synchronous or metachronous metastases and has an estimated age-standardized mortality in Europe of 2.6\% [6]. The major histologic subtypes of RCC is clear-cell RCC (ccRCC, 75-85\%), of which more than one tenth patients would occur fatal recurrence within 5 years after traditional partial or radical nephrectomy. Although pathologic factors such as coagulative necrosis, metastatic status, sarcomatoid features, and lymphovascular invasion (LVI) have been extensively addressed, their impacts on ccRCC prognosis are however inconclusive [7]. Following surgery for localised RCC some recent studies rely on models combining standard models with molecular biomarkers to make progress in better stratifying recurrence risk to aid patient counselling, personalize follow-up, and target adjuvant treatment trails $[8,9]$. Herein sustained studies to evaluate the prognostic value of genetic and proteome signature in RCC patients are unremittingly on going.

Previous works by Evgeniy et al have indicated that $\mathrm{CCR}^{+}$myeloid cell subset is expanded in RCC patients and that their accumulation composes cancer-related inflammation and could contribute to tumor cells immune evasion during metastatic cascade by means of Treg cells aggregation. Furthermore, blockage of CCL1-CCR8 signals may provide an attractive strategy for potential therapeutic intervention [10]. In view of the likelihood of CCR8 expression on tumor cells as it was reported to trigger tumor cells migration or invasion through lymphatic vasculature, and the open data from The Cancer Genome Atlas that CCR8 mRNA are unregulated in $16 \%$ ccRCC cases (61 of 392 ), we hypothesized that intratumoral overexpression of CCR8 may serve as a potential prognosticator of ccRCC recurrence.

In this study, we analyzed the expression of intratumoral CCR8 and the impact of CCR8 expression on recurrence-free survival (RFS) in a large cohort of ccRCC patients without synchronous metastatic diseases.

\section{RESULTS}

\section{Clinical and pathologic characteristic}

After applying initial exclusion criteria, a total of 472 patients with pT1-3N0M0 ccRCC underwent nephrectomy from 2008 to 2009. Mean age in the entire
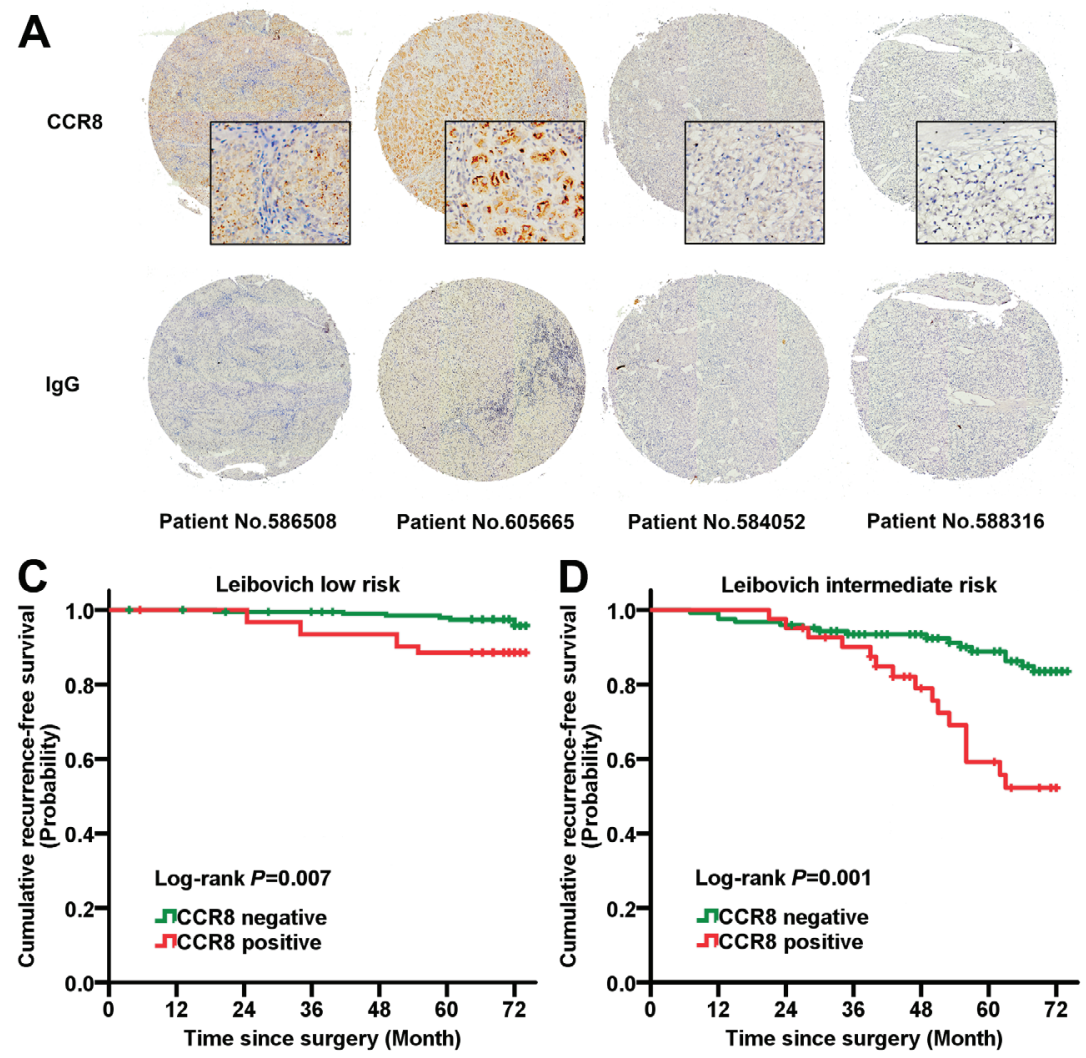
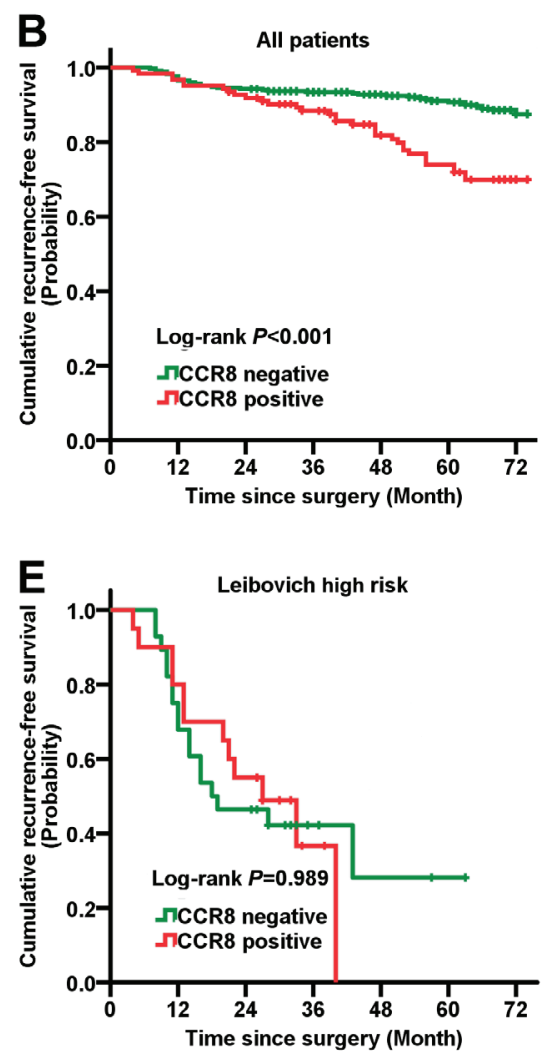

Figure 1: Prognostic power of CCR8 in diverse Leibovich risk groups. A. Typical immunohistochemistry staining images of CCR8 and isotype IgG in ccRCC tumor tissues. B. Kaplan-Meier analysis of RFS in entire ccRCC patients according to intratumoral CCR8 expression. C.-E. Kaplan-Meier analysis of RFS according to intratumoral CCR8 expression in C. Leibovich low risk, D. Leibovich intermediate risk, E. Leibovich high risk patients. Abbreviation: RFS, recurrence-free survival; CCR8, CC chemokine receptor 8. 
Table 1: Correlations between CCR8 expression and clinical characteristics in non-metastatic ccRCC patients

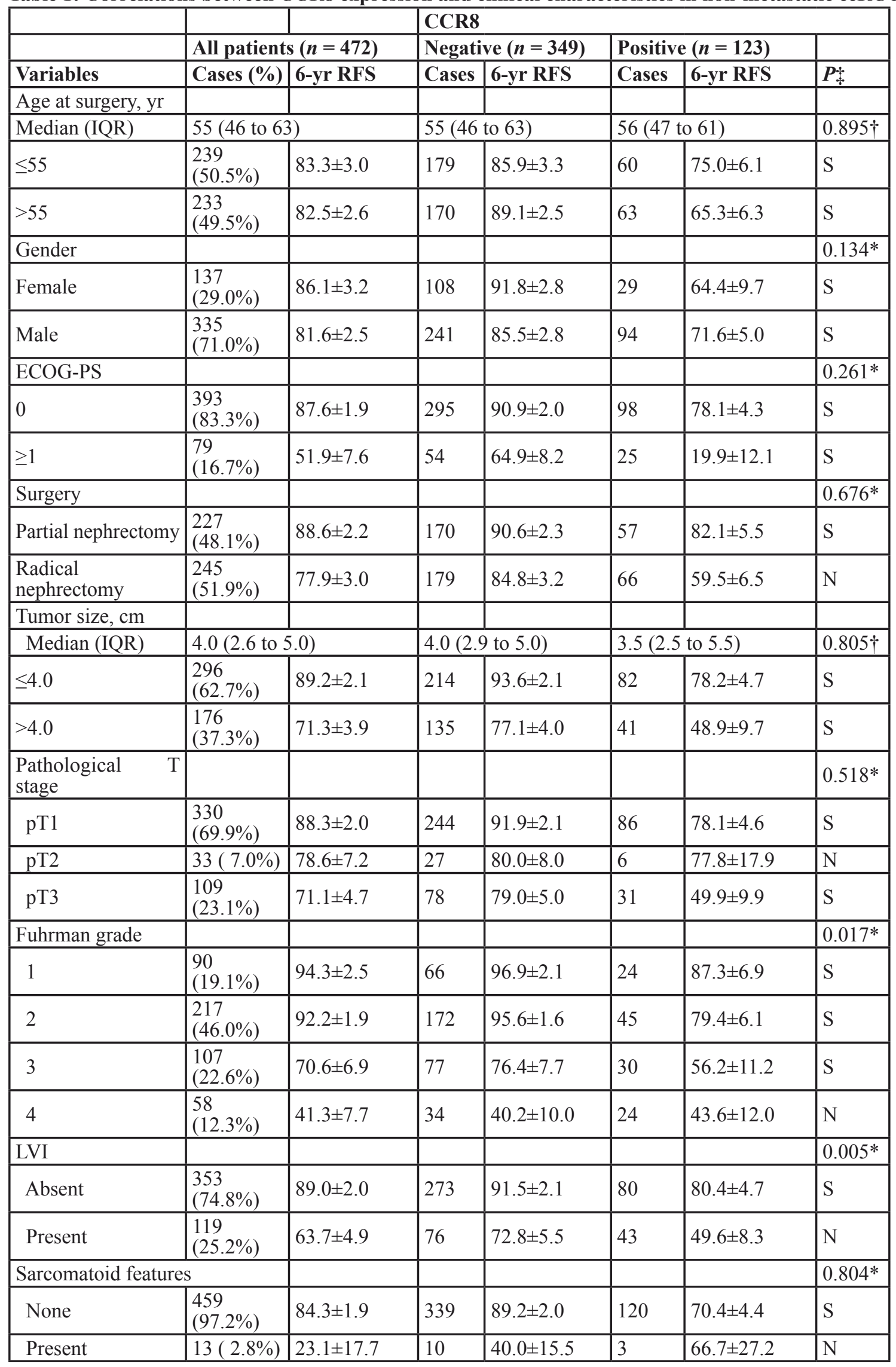




\begin{tabular}{|l|l|l|l|l|l|l|l|}
\hline Coagulative necrosis & & & & & & $0.002^{*}$ \\
\hline None & $\begin{array}{l}377 \\
(79.9 \%)\end{array}$ & $88.5 \pm 1.9$ & 291 & $90.8 \pm 2.1$ & 86 & $81.1 \pm 4.4$ & $\mathrm{~S}$ \\
\hline Present & $\begin{array}{l}95 \\
(20.1 \%)\end{array}$ & $58.9 \pm 5.7$ & 58 & $70.8 \pm 6.3$ & 37 & $38.1 \pm 9.6$ & $\mathrm{~S}$ \\
\hline $\begin{array}{l}\text { Follow-up duration, } \\
\text { mo }\end{array}$ & & & & & & \\
\hline Median (IQR) & $73.0(72.0$ to 74.0$)$ & \multicolumn{7}{|l|}{$73.0(72.0$ to 74.0$)$} & $72.0(71.0$ to 73.0$)$ & $0.092 \dagger$ \\
\hline
\end{tabular}

Abbreviation: CCR8, CC chemokine receptor 8; RFS, recurrence-free survival; ccRCC, clear-cell renal cell carcinoma; ECOG-PS, Eastern cooperative Oncology Group performance status; LVI, lymphovascular invasion; IQR, interquartile range; $\mathrm{S}$, significant (Log-rank test $P<0.05$ ); N, non-significant (Log-rank test $P \geq 0.05$ ).

Outcome estimation is limited to the largest survival time when it is censored.

*Fisher's exact test to assess the correlation between variables and CCR8.

$\dagger$ Wilcoxon rank-sum test.

\$Log-rank test of equality of survival distributions for the different levels of CCR8.

Table 2: Proportional hazard model for RFS prediction of non-metastatic ceRCC patients

\begin{tabular}{|c|c|c|c|c|}
\hline \multirow[b]{2}{*}{ Variables } & \multicolumn{2}{|l|}{ Base model } & \multicolumn{2}{|c|}{ Bootstrap validate model $^{*}$} \\
\hline & HR (95\% CI) & $P$ & HR $(95 \%$ CI) & $P$ \\
\hline Age at surgery (Continuous by 5 -yr increment) & $1.090(0.973$ to 1.222$)$ & 0.137 & Adjusted & \\
\hline Gender (Male $v s$ Female) & $1.559(0.876$ to 2.777$)$ & 0.131 & Adjusted & \\
\hline ECOG-PS $(\geq 1$ vs 0$)$ & $1.310(0.671$ to 2.556$)$ & 0.429 & $1.444(0.592$ to 3.456$)$ & 0.426 \\
\hline Tumor size (Continuous, cm) & $1.358(1.220$ to 1.511$)$ & $<0.001$ & $1.375(1.183$ to 1.613$)$ & 0.003 \\
\hline Pathological T stage (pT1 vs pT2 vs pT3) & & $<0.001$ & & 0.001 \\
\hline pT2 vs pT1 & $2.034(0.815$ to 5.076$)$ & 0.128 & $1.724(0.496$ to 5.501$)$ & 0.226 \\
\hline pT3 vs pT1 & $4.022(2.042$ to 7.920$)$ & $<0.001$ & $3.906(1.428$ to 7.996$)$ & 0.003 \\
\hline Fuhrman grade $(1+2$ vs 3 vs 4$)$ & & $<0.001$ & & 0.001 \\
\hline $3 v s 1+2$ & $2.206(1.152$ to 4.224$)$ & 0.017 & $2.300(1.058$ to 4.821$)$ & 0.018 \\
\hline $4 v s 1+2$ & $4.038(1.994$ to 8.180$)$ & $<0.001$ & $3.985(1.602$ to 9.422$)$ & 0.001 \\
\hline LVI (Present $v s$ Absent) & $2.943(1.742$ to 4.969$)$ & $<0.001$ & $2.727(1.366$ to 5.124$)$ & 0.002 \\
\hline Sarcomatoid features (Present $v s$ Absent) & $5.442(2.060$ to 14.374$)$ & 0.001 & $6.250(1.000$ to 37.115$)$ & 0.042 \\
\hline Coagulative necrosis (Present $v s$ Absent) & 2.724 (1.566 to 4.739$)$ & $<0.001$ & $2.582(1.361$ to 4.993$)$ & 0.002 \\
\hline CCR8 (Positive vs Negative) & $2.014(1.224$ to 3.315$)$ & 0.006 & $2.198(1.154$ to 4.154$)$ & 0.008 \\
\hline
\end{tabular}

Abbreviation: CCR8, CC chemokine receptor 8; RFS, recurrence-free survival; ccRCC, clear-cell renal cell carcinoma; ECOG-PS, Eastern cooperative Oncology Group performance status; LVI, lymphovascular invasion.

*The bootstrap validate model is calculated on the basis of adjusted survival function for age and gender by the time of surgery. Bootstrapping with 1000 resamples were used.

group was $55.1 \mathrm{yr}$ (range 21-86). Of these patients, 123 patients $(26.1 \%)$ had positive CCR8 tissue staining (Figure 1A). Table 1 compares their clinical and pathologic features. Overall, no significant difference of age and gender between $\mathrm{CCR}^{+}$and CCR8 $8^{-}$groups, while higher Fuhrman grade, presence of LVI and necrosis were significantly associated with positive CCR8 expression $(P$ $=0.017,0.005,0.002$, respectively).

\section{Clinical outcomes and association of CCR8 expression with survival}

Median follow-up for patients alive at last followup was 73 months (IQR 72-74, range 39-74, $n=410$ ). 71 patients $(15.0 \%)$ recurred during the follow-up including 54 patients $(11.4 \%)$ who died of RCC. Overall 6-year OS was $86.1 \%(95 \% \mathrm{CI}, 82.9-89.3)$ and RFS was $83.0 \%$
(95\%CI, 79.0-87.0).

Six-year RFS estimates of CCR8 $8^{+}$and CCR8 patients were $69.9(95 \% \mathrm{CI}, 61.3-78.5)$ and $87.5(95 \% \mathrm{CI}$, 83.4-91.6), respectively (Table 1). In univariate analysis, CCR8 positive expression was significantly associated with worse RFS $(P<0.001$; Figure 1B), this difference in survival remained significant when restricting analyses to grade 1-2, negative LVI or negative sarcomatoid patients. Furthermore, positive CCR8 expression was an independent predictor of RFS (HR, 2.014; 95\%CI, $1.224-3.315 ; P=0.006)$ in multivariate analysis. After a 1000-resampled bootstrap correction, it remained its significance (HR, 2.198; 95\%CI, 1.154-4.154; $P=0.008$ ), together with tumor size, pT stage, Fuhrman grade, LVI and coagulative necrosis (Table 2). 
Table 3: Prognostication comparison of built-up nomogram and original Leibovich model

\begin{tabular}{|c|c|c|c|c|c|c|c|}
\hline & & C-index (95\%CI & & & & AIC & \\
\hline Patients group & \begin{tabular}{|l} 
No. of \\
patients $(\%)$
\end{tabular} & Nomogram & Leibovich & \begin{tabular}{|l|}
$\begin{array}{l}\text { Coefficient } \\
(95 \% \text { CI })\end{array}$ \\
\end{tabular} & $P$ & Nomogram & Leibovich \\
\hline Overall & $472(100 \%)$ & $\begin{array}{l}0.854(0.811 \text { to } \\
0.896)\end{array}$ & $\begin{array}{l}0.836(0.790 \text { to } \\
0.882)\end{array}$ & $\begin{array}{l}0.037 \text { (0.013 to } \\
0.061)\end{array}$ & 0.010 & 706.0 & 738.2 \\
\hline TNM stage I+II & $345(73.1 \%)$ & $\begin{array}{l}0.819(0.750 \text { to } \\
0.888)\end{array}$ & \begin{tabular}{|l}
$0.768(0.696$ to \\
$0.839)$
\end{tabular} & $\begin{array}{l}0.051 \quad(0.001 \text { to } \\
0.101)\end{array}$ & 0.044 & 404.8 & 410.0 \\
\hline $\begin{array}{l}\text { UISS low or } \\
\text { intermediate risk }\end{array}$ & $438(92.8 \%)$ & $\begin{array}{l}0.847 \quad(0.790 \text { to } \\
0.903)\end{array}$ & $\begin{array}{l}0.797(0.738 \text { to } \\
0.856)\end{array}$ & $\begin{array}{l}0.048(0.015 \text { to } \\
0.082)\end{array}$ & 0.005 & 559.7 & 593.9 \\
\hline $\begin{array}{l}\text { SSIGN low or } \\
\text { intermediate risk }\end{array}$ & $458(97.0 \%)$ & $\begin{array}{l}0.848 \text { (0.796 to } \\
0.900)\end{array}$ & $\begin{array}{l}0.801(0.746 \text { to } \\
0.856)\end{array}$ & $\begin{array}{l}0.049(0.018 \text { to } \\
0.079)\end{array}$ & 0.002 & 608.5 & 645.0 \\
\hline
\end{tabular}

Abbreviation: C-index, concordance index; AIC, Akaike's information criterion; UISS, UCLA Integrated Staging System; SSIGN, Mayo clinic stage, size, grade, and necrosis; 95\% CI, 95\% confidence interval.

C-index, 95\%CI and AIC are calculated from 1000 bootstrap samples to protect from overfitting.

\section{Predictive impact of CCR8 upon Leibovich score model}

The Leibovich recurrence risk scores of all 472 patients were calculated and divided into three risk groups: low risk (score $0-2 ; n=260,55.1 \%$ ), intermediate risk (score $3-5 ; n=164,34.7 \%$ ), high risk (score $\geq 6 ; n=48$, $10.2 \%$ ). Kaplan-Meier survival analyses revealed that the diverse outcome between $\mathrm{CCR} 8^{+}$and $\mathrm{CCR} 8^{-}$patients was dominantly lay in Leibovich low and intermediate risk groups (Log-rank $P=0.001,0.007$, respectively; Figure $1 \mathrm{C}-1 \mathrm{E})$. Stratified multivariate analyses also showed an independent predictive impact of CCR8 for RFS in Leibovich low $(\mathrm{HR}=4.616 ; P=0.009)$ and intermediate risk groups $(\mathrm{HR}=4.002 ; P=0.006)$. These data manifested the significant RFS prognoses power of CCR8 in low recurrence risk population of localized ccRCC patients and that this indication was differ from those risk factors composing Leibovich model, including pT stage, Fuhrman grade, tumor size, and necrosis (Figure 2).

\section{Construction and validation of prognostic nomogram for RFS}

A nomogram that incorporated the significant prognostic factors concluded from validated multivariate analyses was established (Table 2 and Figure 3A). Considering the wide variation on confidence interval of sarcomatoid feature, which may resulted from their few effective events, we excluded sarcomatoid feature in the final nomogram without compromising the robustness of the model. The nomogram illustrated pathological $\mathrm{T}$ stage, Fuhrman grade and tumor size as sharing the largest contribution to prognostication (weighted ratio $=0.189$, $0.237,0.227$, respectively). Intratumoral CCR8 expression showed a moderate impact on outcome (weight ratio $=$ 0.071). Each level of these variables was assigned a score on the scale, and the total score could be easily used to determine the estimated probability of survival at different time point.

The calibration plots presented an excellent agreement in the cohort and a condign consistency in bootstrap resampling analysis between the predicted and actual observation for 3-yr and 6-yr RFS (3-yr RFS data not shown; Figure 3B). In the primary cohort, the Harrell's C-index for the established nomogram to predict RFS (0.854; 95\%CI, 0.811-0.896) was significant higher than that of the Leibovich model $(0.836 ; 95 \%$ CI, 0.790-0.882; $P=0.010)$, and the AIC was also lower than Leibovich model (706.0 vs 738.2). This superior performance of nomogram was also kept significant among TNM I+II patients, UISS model defined and SSIGN score defined low-intermediate risk patients $(P=0.044,0.005,0.002$, respectively; Table 3 ).

\section{DISCUSSION}

To date $>5600$ separate reports related to $\mathrm{RCC}$ outcome prediction have been published. Unfortunately, the prognostic assessment remains controversial and no remarkable progress was made on validating and applying new parameters in routine clinical practice [7]. Many recent prospective studies screened and reported molecular signatures to join existing prognostic models, which represent underlying dysregulation of biological functions associated with disease aggressiveness, including immune response $[8,11,12]$. A good way to select these prognostic factors representing cancer-related immune response may be considering the biological functions of chemokines or cytokines and their sources or targeting cell types, which could help us to subgroup these factors functionally and then select appropriate agents according to their performance.

Chemokine (C-C motif) receptor (CCR8) is one of the novel candidates of tumor prediction because there have been various literatures reported the special behaviors of CCR8 in different pathogenesis. CCR8 was reported to be expressed on Treg cells which may trigger immune inhibition in several inflammatory diseases 
[14]. Furthermore, evidence rose that endothelialderived spindle cells of human Kaposi sarcoma acquire chemotaxis through its intrinsic CCR8 expression [15].
The only report that directly indicated the pro-tumorigenic expression of CCR8 on tumor cells was conducted by Das et al, which showed that blocking CCR8 function could

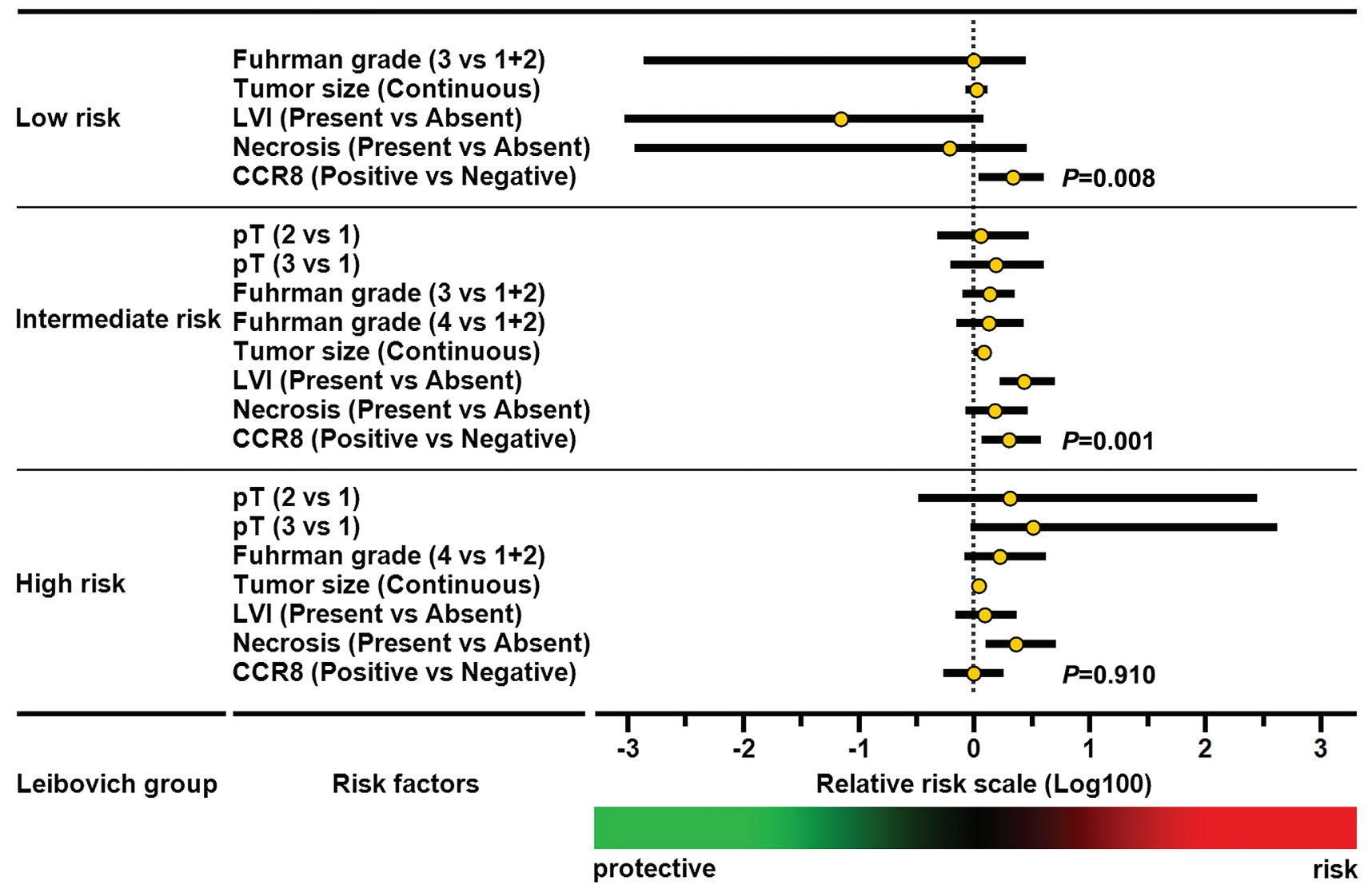

Figure 2: Multivariate analyses of conventional prognostic features in diverse Leibovich risk groups. The relative hazard of each feature for recurrence are scaled in logarithmic form, $P$-values of CCR8 expression pertinence to recurrence-free survival are highlighted. Abbreviation: LVI, lymphovascular invasion; RFS, recurrence-free survival; CCR8, CC chemokine receptor 8.

A

Risk points

Pathological T stage

Fuhrman grade

Tumor size

Coagulative necrosis

LVI

CCR8

Total recurrence risk points

3-Year RFS Probability
6-Year RFS Probability
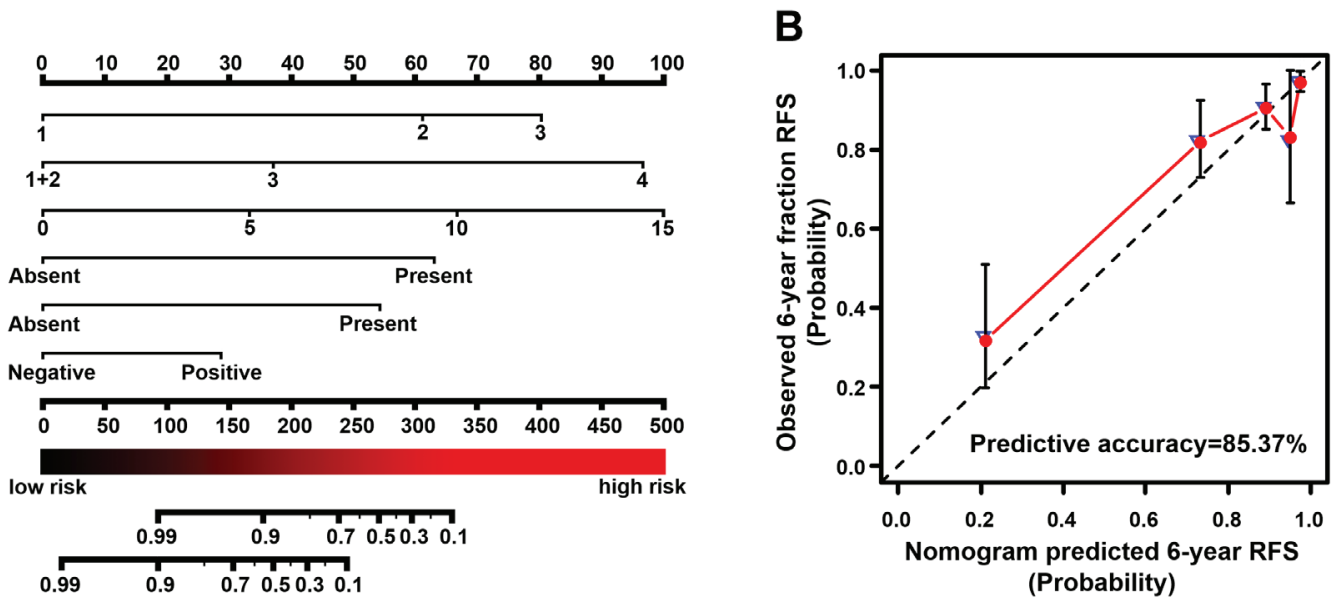

B

Figure 3: Built-up prognostic nomogram and calibration plots for RFS prediction of postoperative ccRCC patients.
A. Six independent prognostic factors including pathological T stage, Fuhrman grade, tumor size, coagulative necrosis, LVI presentation and CCR8 expression were identified and entered into the nomogram to predict 6-year recurrence risk and recurrence-free survival. B. Calibration curves for predicting 6-year RFS of ccRCC patients. The nomogram-predicted RFS is plotted on the x-axis, actual RFS (solid red circles) and bootstrapped RFS (blue hollow triangle) are plotted on the y-axis, a plot along the 45-degree (dash line) indicate a perfect calibration in which the predicted probabilities are identical to the actual outcomes. Abbreviation: LVI, lymphovascular invasion; RFS, recurrence-free survival; CCR8, CC chemokine receptor 8 . 
inhibits CCR8+ melanoma cell egress from afferent lymphatic into lymph node [5]. However, the expression of CCR8 in RCC remained unclear, despite a recent work showed that up-regulated expression of CCR8 is detected within RCC tissues and primarily limited to CD11 $\mathrm{b}^{+}$tumor associated macrophages, while their work enrolled limited 22 RCC patients [10].

In this study, we detected the expression in tumor cells from a segment of non-metastatic ccRCC cases detected and confirmed the intratumoral CCR 8 expression in $26 \%$ (123 of 472) ccRCC tissue samples, comparative with that of $16 \%$ reported by 2013 TCGA cohort data. Analogously, we demonstrated a significant correlation between positive CCR8 expression and the likelihood of post-operative recurrence by competing risk analyses. In order to further strengthen the prognostication impact of CCR8 for clinical recurrence, we developed a postoperative nomogram to predict long-term recurrence-free survival of non-metastatic patients based on 6-year followup. The built-up model offer further benefit in addition to the current combined clinicopathologic stratification tools - Leibovich score, which is constructed by pathologic T stage, $\mathrm{N}$ stage, tumor size, nuclear grade and histologic necrosis [17]. Of particular interest, we found that this improvement mainly occurred in organ-confined patients or low risk patients defined by UISS and SSIGN models. This may help us to inform postoperative management of early-stage patients for whom the conventional $\mathrm{T}$ stage and Fuhrman grades may have weaken prediction effects, as well as to help recognize the patients with low-risk tumor who would ultimately progress and succumb to their disease as an aid of conventional abdomen imaging techniques [18].

As far as we have found, CCR8 is the only C-C motif family chemokine receptor that is dominantly expressed on renal tumor cells instead of infiltrating immune cells while exerting profound impact on recurrence-free survival in localised patients, which means we can easily judge the IHC staining results by positive/negative dichotomy either artificially or digitally, with few effect from intratumoral heterogeneity and immune cell infiltrating susceptibility. This findings may indicated CCR8 as a potential agent candidate of $\mathrm{C}-\mathrm{C}$ motif chemokine receptor family. It should be acknowledged that this study lack independent external validation, and it seem likely that the study design of retrospectively analyzing post-nephrectomy tumor samples, particularly with the intratumoral heterogeneity of regimens, would hinder the robustness of predictive biomarkers, further experimental studies are also required to identify the detailed role of CCR8 in ccRCC. Nevertheless, we should notice that attempts to identify pharmacological antagonists of CCR8 have been going on, and our work may implicate therapeutic targets of those small molecule inhibitors used on adjuvant therapy for non-metastatic ccRCC [19].

\section{MATERIALS AND METHODS}

\section{Patient selection}

The study database included 472 non-metastatic clear-cell RCC patients from Zhongshan Hospital, Fudan University, Shanghai, China. The primary inclusion criterion were (1) histopathologically-proven clearcell RCC; (2) received partial- or radical- nephrectomy between Jan 7, 2008 and Dec 23, 2009; (3) had available corresponding archived Formalin Fixed Paraffin Embedded (FFPE) specimen of tumor mass $\left(\geq 1 \mathrm{~cm}^{3}\right)$. All these 490 valid patients received surgery after diagnoses, without pre-surgery radiotherapy or chemotherapy. Seven T4 stage patients, seven M1 metastatic patients, and four failed-to-stain cases were then excluded from the cohort, leaving 472 final cases. To ensure consistent data collection, baseline demographic, clinical, and laboratory data were collected simultaneously, MRI and CT scans were reassessed in radiology units, and all archived diagnostic H\&E slides were pathologically central reviewed by pathologist (Chen L.) independently. This study was approved by the institutional ethical review boards of hospital and all patients stated informed consent along with phone-call follow-up.

\section{Data collection}

The primary outcome of interest was recurrence-free survival (RFS). RFS was defined as the time from surgery to first renal cell carcinoma recurrence (local or distant metastases, identified by imaging, biopsy, or physical examination). According to clinicians and archived files, occurrences were censored if patients died without clear evidence of recurrence or if the patient was alive at end of follow-up. Follow-up data for all patients were obtained from most recent medical review and ended in Mar 2015. All patients were examined routinely every 5-6 months during the first 5 years of follow-up and annually thereafter. The study did not include any patients with recurrence within 2 months of surgery.

All relevant information on sociodemographic data (age at surgery, gender), pathologic data (pathologic tumor, node, and metastasis status), treatment-related data (type of nephrectomy or chemotherapy). Histological subtypes were re-stratified according to 2014 EAU guidelines [6]. TNM staging was re-classified according to 2010 AJCC TNM classification [20]. Fuhrman grade, LVI, coagulative necrosis, sarcomatoid features were reported according to 2012 ISUP consensus [7]. Eastern ECOG-PS were prospectively recorded and re-archived as previously described [21]. UISS, SSIGN and Leibovich scores were applied to all valid patients according to original scoring algorithm, respectively $[17,22,23]$. 


\section{Statistical analysis}

Associations between CCR8 expression subgroups versus clinicopathologic parameters were evaluated using Fisher exact test and Wilcoxon rank-sum test. RFS was assessed and graphically illustrated using Kaplan-Meier and log-rank test was used for comparing different scoring categories. Independent associations between RFS and assessed clinicopathologic predictors were evaluated using multivariate Cox proportional hazards regression models. Prognostic parameters that were found to be associated with recurrence risk in the development study were further narrowed down to form a nomogram by the strength of the associations with recurrence in both univariate and multivariate analyses. The concordance index (C index) and Akaike's Information Criteria (AIC) were used to assess the predictive accuracy and sufficiency of different models, while Hanley-McNeil test was used to compare between $\mathrm{C}$ index [24, 25]. To reduce overfit bias and internally validate the predictive accuracy estimates, multivariable models and $\mathrm{C}$ index calculations were subjected to 1000 bootstrap resamples.

Statistical analyses were performed with SPSS, version 21.0 (IBM, Armonk, NY), Stata SE, version 12.1 (Stata, College Station, TX) and R software packages, version 3.1.2 (The R Foundation for Statistical Computing, http://www.r-project.org/). A two-sided $p$ value of less than 0.05 was considered to be statistically significant for all reports.

\section{Procedures}

Primary FFPE RCC samples were obtained from the Department of Urology, Zhongshan Hospital with patients' consent and approval of the institutional review board of Fudan University. Microarray development and immunohistochemistry were performed according to the methods previously applied [26] with appropriate antibodies after control staining (anti-CCR8 antibody, ab140796, Abcam, diluted 1/100) (Supplementary Document S1). Immunohistochemistry sections and corresponding $\mathrm{H} \& \mathrm{E}$ sections were scanned by a fully automated microscopy system (Leica DM6000 B, Leica Microsystems GmbH, Mannheim, Germany), images were captured by Leica CV-M2CL camera and analyzed by Leica Ariol 4.0 software automatically. Cases were considered positive for expression when $>10 \%$ of tumor cells showed diffuse immunoreactivity.

\section{CONFLICTS OF INTEREST}

The authors declare no competing interests.

\section{GRANT SUPPORT}

This study was funded by grants from National Basic Research Program of China (2012CB822104), National Natural Science Foundation of China (31100629, 31270863, 81471621, 81472227, 81402082 and 81402085), Program for New Century Excellent Talents in University (NCET-13-0146) and Shanghai Rising-Star Program (13QA1400300). All these study sponsors have no roles in the study design, in the collection, analysis, and interpretation of data.

\section{REFERENCES}

1. Louahed J, Struyf S, Demoulin JB, Parmentier M, Van Snick J, Van Damme J and Renauld JC. CCR8-dependent activation of the RAS/MAPK pathway mediates antiapoptotic activity of I-309/ CCL1 and vMIP-I. European journal of immunology. 2003; 33:494-501.

2. Denis C, Deiteren K, Mortier A, Tounsi A, Fransen E, Proost P, Renauld JC and Lambeir AM. C-terminal clipping of chemokine CCL1/I-309 enhances CCR8-mediated intracellular calcium release and anti-apoptotic activity. PloS one. 2012; 7:e34199.

3. Oshio T, Kawashima R, Kawamura YI, Hagiwara T, Mizutani N, Okada $\mathrm{T}$, Otsubo $\mathrm{T}$, Inagaki-Ohara $\mathrm{K}$, Matsukawa A, Haga T, Kakuta S, Iwakura Y, Hosokawa $\mathrm{S}$ and Dohi T. Chemokine receptor CCR8 is required for lipopolysaccharide-triggered cytokine production in mouse peritoneal macrophages. PloS one. 2014; 9:e94445.

4. Ruckes T, Saul D, Van Snick J, Hermine O and Grassmann R. Autocrine antiapoptotic stimulation of cultured adult T-cell leukemia cells by overexpression of the chemokine I-309. Blood. 2001; 98:1150-1159.

5. Das S, Sarrou E, Podgrabinska S, Cassella M, Mungamuri SK, Feirt N, Gordon R, Nagi CS, Wang Y, Entenberg D, Condeelis $\mathrm{J}$ and Skobe M. Tumor cell entry into the lymph node is controlled by CCL1 chemokine expressed by lymph node lymphatic sinuses. The Journal of experimental medicine. 2013; 210:1509-1528.

6. Ljungberg B, Bensalah $\mathrm{K}$, Canfield S, Dabestani S, Hofmann F, Hora M, Kuczyk MA, Lam T, Marconi L, Merseburger AS, Mulders P, Powles T, Staehler M, Volpe A and Bex A. EAU Guidelines on Renal Cell Carcinoma: 2014 Update. European urology. 2015.

7. Delahunt B, Cheville JC, Martignoni G, Humphrey PA, Magi-Galluzzi C, McKenney J, Egevad L, Algaba F, Moch H, Grignon DJ, Montironi R, Srigley JR and Members of the IRTP. The International Society of Urological Pathology (ISUP) grading system for renal cell carcinoma and other prognostic parameters. The American journal of surgical pathology. 2013; 37:1490-1504.

8. Ko JJ, Xie W, Kroeger N, Lee JL, Rini BI, Knox JJ, Bjarnason GA, Srinivas S, Pal SK, Yuasa T, Smoragiewicz M, Donskov F, Kanesvaran R, Wood L, Ernst DS, Agarwal 
$\mathrm{N}$, et al. The International Metastatic Renal Cell Carcinoma Database Consortium model as a prognostic tool in patients with metastatic renal cell carcinoma previously treated with first-line targeted therapy: a population-based study. The Lancet Oncology. 2015; 16:293-300.

9. Rini B, Goddard A, Knezevic D, Maddala T, Zhou M, Aydin H, Campbell S, Elson P, Koscielny S, Lopatin M, Svedman C, Martini JF, Williams JA, Verkarre V, Radulescu C, Neuzillet Y, et al. A 16-gene assay to predict recurrence after surgery in localised renal cell carcinoma: development and validation studies. The Lancet Oncology. 2015.

10. Eruslanov E, Stoffs T, Kim WJ, Daurkin I, Gilbert SM, Su LM, Vieweg J, Daaka Y and Kusmartsev S. Expansion of CCR8(+) inflammatory myeloid cells in cancer patients with urothelial and renal carcinomas. Clinical cancer research. 2013; 19:1670-1680.

11. Choudhury Y, Wei X, Chu YH, Ng LG, Tan HS, Koh V, Thike AA, Poon E, Ng QS, Toh CK, Kanesvaran R, Tan $\mathrm{PH}$ and Tan $\mathrm{MH}$. A multigene assay identifying distinct prognostic subtypes of clear cell renal cell carcinoma with differential response to tyrosine kinase inhibition. European urology. 2015; 67:17-20.

12. Brooks SA, Brannon AR, Parker JS, Fisher JC, Sen O, Kattan MW, Hakimi AA, Hsieh JJ, Choueiri TK, Tamboli P, Maranchie JK, Hinds P, Miller CR, Nielsen ME and Rathmell WK. ClearCode34: A prognostic risk predictor for localized clear cell renal cell carcinoma. European urology. 2014; 66:77-84

13. Chensue SW, Lukacs NW, Yang TY, Shang X, Frait KA, Kunkel SL, Kung T, Wiekowski MT, Hedrick JA, Cook DN, Zingoni A, Narula SK, Zlotnik A, Barrat FJ, O'Garra A, Napolitano M, et al. Aberrant in vivo T helper type 2 cell response and impaired eosinophil recruitment in $\mathrm{CC}$ chemokine receptor 8 knockout mice. The Journal of experimental medicine. 2001; 193:573-584.

14. Coghill JM, Fowler KA, West ML, Fulton LM, van Deventer H, McKinnon KP, Vincent BG, Lin K, Panoskaltsis-Mortari A, Cook DN, Blazar BR and Serody JS. CC chemokine receptor 8 potentiates donor Treg survival and is critical for the prevention of murine graftversus-host disease. Blood. 2013; 122:825-836.

15. Haque NS, Fallon JT, Taubman MB and Harpel PC. The chemokine receptor CCR8 mediates human endothelial cell chemotaxis induced by I-309 and Kaposi sarcoma herpesvirus-encoded vMIP-I and by lipoprotein(a)stimulated endothelial cell conditioned medium. Blood. 2001; 97:39-45.

16. Angelova M, Charoentong P, Hackl H, Fischer ML, Snajder R, Krogsdam AM, Waldner MJ, Bindea G, Mlecnik B, Galon J and Trajanoski Z. Characterization of the immunophenotypes and antigenomes of colorectal cancers reveals distinct tumor escape mechanisms and novel targets for immunotherapy. Genome biology. 2015; 16:64.

17. Leibovich BC, Blute ML, Cheville JC, Lohse CM, Frank I,
Kwon ED, Weaver AL, Parker AS and Zincke H. Prediction of progression after radical nephrectomy for patients with clear cell renal cell carcinoma: a stratification tool for prospective clinical trials. Cancer. 2003; 97:1663-1671.

18. Kroeger N, Zimmermann U, Burchardt M and Pantuck AJ. Prognostication in localised renal cell carcinoma. The Lancet Oncology. 2015.

19. Ghosh S, Elder A, Guo J, Mani U, Patane M, Carson K, Ye Q, Bennett R, Chi S, Jenkins T, Guan B, Kolbeck R, Smith S, Zhang C, LaRosa G, Jaffee B, et al. Design, synthesis, and progress toward optimization of potent small molecule antagonists of CC chemokine receptor 8 (CCR8). Journal of medicinal chemistry. 2006; 49:2669-2672.

20. Kim SP, Alt AL, Weight CJ, Costello BA, Cheville JC, Lohse C, Allmer C and Leibovich BC. Independent validation of the 2010 American Joint Committee on Cancer TNM classification for renal cell carcinoma: results from a large, single institution cohort. The Journal of urology. 2011; 185:2035-2039.

21. Karakiewicz PI, Trinh QD, de la Taille A, Abbou CC, Salomon L, Tostain J, Cindolo L, Artibani W, Ficarra V and Patard JJ. ECOG performance status 0 or 1 and symptom classification do not improve the ability to predict renal cell carcinoma-specific survival. European journal of cancer. 2007; 43:1023-1029.

22. Zisman A, Pantuck AJ, Wieder J, Chao DH, Dorey F, Said JW, deKernion JB, Figlin RA and Belldegrun AS. Risk group assessment and clinical outcome algorithm to predict the natural history of patients with surgically resected renal cell carcinoma. Journal of clinical oncology. 2002; 20:45594566.

23. Frank I, Blute ML, Cheville JC, Lohse CM, Weaver AL and Zincke $\mathrm{H}$. An outcome prediction model for patients with clear cell renal cell carcinoma treated with radical nephrectomy based on tumor stage, size, grade and necrosis: the SSIGN score. The Journal of urology. 2002; 168:23952400.

24. Harrell FE, Jr., Lee KL and Mark DB. Multivariable prognostic models: issues in developing models, evaluating assumptions and adequacy, and measuring and reducing errors. Statistics in medicine. 1996; 15:361-387.

25. Hanley JA and McNeil BJ. A method of comparing the areas under receiver operating characteristic curves derived from the same cases. Radiology. 1983; 148:839-843.

26. Xu L, Zhu Y, An H, Liu Y, Lin Z, Wang G and Xu J. Clinical significance of tumor-derived IL-1beta and IL18 in localized renal cell carcinoma: Associations with recurrence and survival. Urologic oncology. 2014. 